\title{
Dysphagia and thoracoabdominal aneurysm
}

\author{
C W Taylor, A Sinha, J M D Nightingale
}

\begin{abstract}
Two elderly patients who presented with gradually progressive dysphagia are described. Investigations excluded an intraluminal obstruction and showed extrinsic compression of the oesophagus by an aneurysmal aorta. Surgery was not performed and they were successfully managed with a liquid diet.

(Postgrad Med f 2001;77:257-258)
\end{abstract}

Keywords: dysphagia; aortic aneurysm; vascular compression

Dysphagia in the elderly can arise from benign or neoplastic strictures, neuromuscular disorders, and extrinsic compression of the oesophagus. Extrinsic compression by a vascular structure is uncommon. Usually this is due to an aberrant subclavian artery but rarely can be caused by the aorta. ${ }^{1}$ We present two such cases.

\section{Case reports}

CASE 1

An 86 year old women, who was on treatment for hypertension, presented with a six month history of dysphagia for liquids and solids. She had no pain or anorexia but she had lost $5 \mathrm{~kg}$ in weight. She had a pulsatile epigastric mass. Her chest radiograph showed a widened mediastinum. Upper gastrointestinal endoscopy showed a narrow pulsatile lower oesophageal lumen through which the scope easily passed. A barium swallow showed a dilated tortuous oesophagus with narrowing at the cardia giving

\section{Department of Gastroenterology, Leicester Royal Infirmary, Leicester, UK \\ C W Taylor \\ A Sinha \\ J M D Nightingale \\ Correspondence to: Dr A Sinha, \\ Gastroenterology Centre, \\ Leicester Royal Infirmary, \\ Infirmary Square, Leicester \\ LE1 5WW, UK \\ asinha@uhl.trent.nhs.uk}

Submitted 11 May 2000 Accepted 20 September 2000

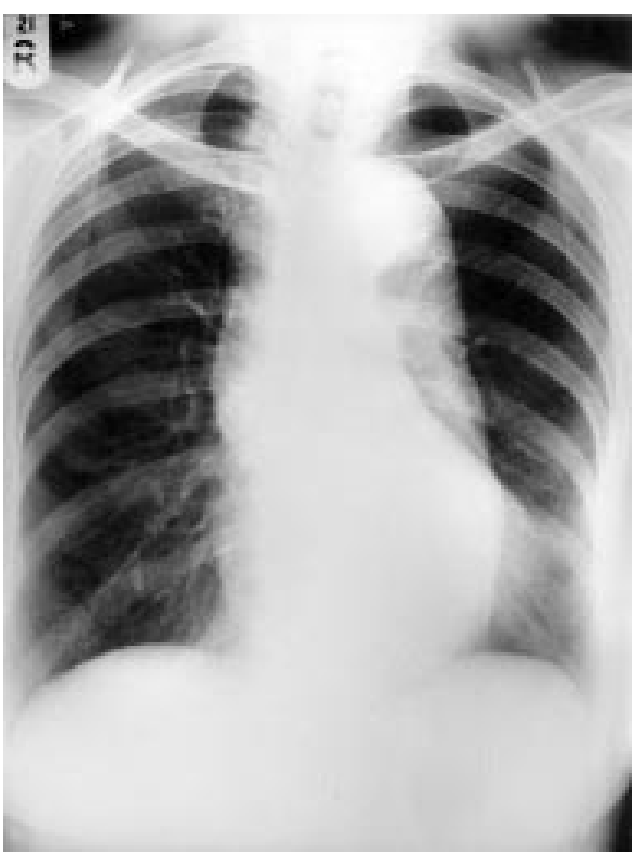

Figure 1 Chest radiograph in case 2. the classical appearance of achalasia. Oesophageal manometry showed propagated peristalsis of low amplitude but the tube would not cross the cardia. Abdominal ultrasound and computed tomography confirmed a large abdominal aortic aneurysm extending from the chest and into the mid-abdomen with a maximum diameter of $6 \mathrm{~cm}$; it compressed the oesophagus above the gastro-oesophageal junction.

The patient was not considered appropriate for surgical treatment and was given a liquidised diet. She was well a year after diagnosis.

\section{CASE 2}

An 81 year old women, on antihypertensive treatment, presented with a two month history of dysphagia and loss of appetite. A chest radiograph showed a widened mediastinum (fig 1). A barium swallow showed narrowing of the lower oesophageal lumen (fig 2). Oesophageal manometry showed low amplitude waves and a pulsatile high pressure area in the lower oesophagus, which relaxed poorly in response to swallows. Abdominal ultrasound and magnetic resonance imaging (MRI) showed the aorta to be aneurysmal along its entire length with a maximum diameter of $5.5 \mathrm{~cm}$ and compressing the lower oesophagus.

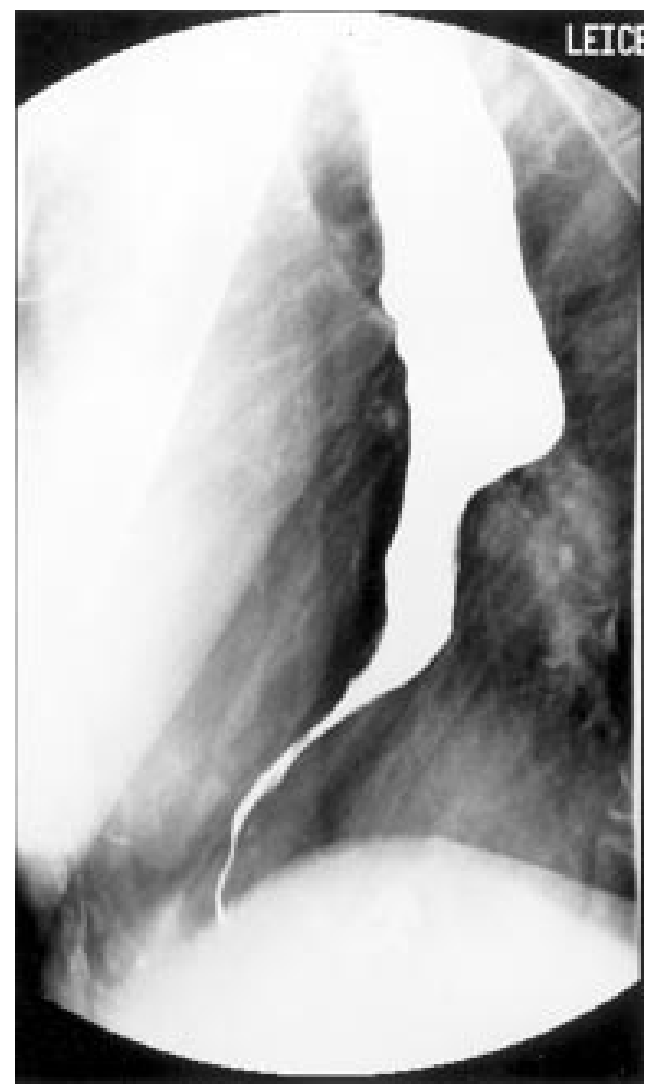

Figure 2 Barium swallow in case 2. 
She was not considered fit for surgery and was managed on a liquidised diet. Two years later she had lost $2 \mathrm{~kg}$ in weight but was otherwise well.

\section{Discussion}

The oesophagus is normally located on the right side of the descending thoracic aorta and crosses over to the left side anterior to the aorta about $5 \mathrm{~cm}$ above the diaphragm. ${ }^{2}$ Dysphagia aortica can be caused by compression of either the upper oesophagus by a thoracic aortic aneurysm or the lower oesophagus by an aneurysmal or atherosclerotic aorta. It is associated with hypertension, old age, and kyphoscoliosis. ${ }^{3}$ Only eight cases of dysphagia aortica were identified from a Medline search of the last 10 years and review of previous literature compared with 40 cases of compression by an aberrant subclavian artery.

When a patient has dysphagia and an aortic aneurysm, the diagnosis of dysphagia aortica is made on the basis of barium swallow and manometry. The barium swallow will show classical feature of achalasia, that is a dilated atonic oesophagus with a narrow tapered point at the cardia. Manometry, which differentiates dysphagia aortica from achalasia, shows low amplitude propagated peristaltic waves in the proximal part of the oesophagus and a high pressure band at the site of vascular compression. In addition there may be superimposed pulsations in the pressure wave coinciding with the cardiac cycle. This contrasts with true achalasia in which there are no propagated contractions and no superimposed pulsations. Endoscopy may reveal pulsatile extrinsic compression or stenosis of the lower oesophagus with proximal dilatation. Other imaging techniques that may be useful in diagnosis include chest radiography, computed tomography, and MRI. It is important to remember that diffusely infiltrating adenocarcinomas arising from the gastro-oesophageal junction can mimic the radiological and manometric features of true achalasia, by mechanical obstruction of the distal oesophagus as well as by infiltration and destruction of the myenteric plexus by the tumour. ${ }^{4}$ This condition, termed pseudoachalasia, must be excluded before making a diagnosis of either classical achalasia

\section{Learning points}

- Thoracoabdominal aortic aneurysms may cause dysphagia.

- Barium swallow appears identical to achalasia but manometry shows low amplitude propulsive contractions.

- Surgical intervention is often inappropriate.

or dysphagia aortica. Differentiation from dysphagia aortica is possible on the basis of manometry, which reveals an absence of propagated contractions and superimposed vascular pulsations. There is no single diagnostic test for pseudoachalasia, but high resolution computed tomography, MRI, and endoscopic ultrasound and careful histological examination of adequate endoscopic biopsy specimens from the area can be used to make the diagnosis.

Aortic or aberrant subclavian artery compression of the oesophagus as reflected by manometry does not necessarily correlate with the presence of dysphagia. ${ }^{5-7}$ It is therefore advisable for manometry to be used only when a barium swallow done for dysphagia has suggested achalasia. ${ }^{6}$

As surgery is often inappropriate, conservative management is usually the only option. This consists of treatment of hypertension and heart failure and a change to a semisolid or liquid diet; this provided symptomatic relief to our two patients.

Dysphagia aortica should be considered in any elderly patient with dysphagia who also has an aortic aneurysm.

1 Pape R. Uber einen abnormen verlauf ("tiefe Rechtslage") der mesa aortitischen descendens. Fortschr Roentgenstr 1932;46:257-69.

2 Williams PL, ed. Gray's anatomy. Edinburgh: Churchill Livingstone, 1995 .

3 McMillian IKR, Hyde I. Compression of oesophagus by aorta. Thorax 1969;24:32-8

4 Robertson CS, Griffith CDM, Atkinson M, et al. Pseudoachalasia of the cardia: a review. $\mathcal{F} R$ Soc $M e d$ 1988;81:399-402.

5 Mittal RK, Siskind BN, Hongo M, et al. Dysphagia aortica. Clinical, radiological and manometric findings. Dig Dis Sci 1968;31:379-84.

6 Wilkinson JM, Euinton HA, Smith LF, et al. Diagnostic dilemmas in dysphagia aortica. Eur $\mathcal{f}$ Cardiothoracic Surg dilemmas in dyst

7 Sundaram U, Traube M. Radiologic and manometric study of the gastroesophageal junction in dysphagia aortica. $\mathcal{F}$ Clin of the gastroesophageal junction
Gastroenterol 1995;21:275-8 